\title{
Welz, Martin (2020): Afrika seit der Dekolonisation. Geschichte und Politik
}

\author{
Stuttgart: Kohlhammer. 301 Seiten. 39€
}

\section{Tim Niendorf}

Angenommen: 31. März 2021 / Online publiziert: 26. April 2021

(C) Der/die Autor(en) 2021

Mit „Afrika seit der Dekolonisation“ legt Martin Welz ein neues deutschsprachiges Standardwerk zur Einführung in die Politik des afrikanischen Kontinents vor. Die auf den ersten Blick noch recht klassisch strukturierte Monografie unterteilt sich in zwölf Einzelkapitel, wobei sich das erste Drittel mit den politischen Entwicklungen seit dem sogenannten „Goldenen Zeitalter“, insbesondere aber von der Kolonisation bis zu den 2010er-Jahren beschäftigt. Die anschließenden zwei Kapitel behandeln die Wirtschaftsentwicklung, während sich das siebte Kapitel mit der Struktur der politischen Systeme im engeren Sinne auseinandersetzt. Das letzte Drittel gliedert sich in zwei größere, engverknüpfte Problemkomplexe, nämlich Krisen und Konflikte sowie deren Lösung und der Außenpolitik afrikanischer Staaten sowohl untereinander als auch in der überkontinentalen internationalen Politik. Zentral für die Beurteilung des Buchs ist die vom Autor gewählte Zielstellung, ein möglichst umfassendes Bild der Diversität des Kontinents zu zeichnen, gleichzeitig aber den Fokus auf die ,großen Fragen“ zu legen (S. 9-10). Um dies zu erreichen, sollen sowohl die teilweise diametral entgegengesetzten Interpretationen, die Afrika entweder als Kontinent endloser Probleme oder ein kommendes „Afrotopia“ sehen (S. 13), als auch daraus folgende verallgemeinernde Einschätzungen zur Entwicklung des Kontinents hinterfragt werden. Ein tiefergehendes Verständnis der Diversität Afrikas kann demnach mit der Lektüre des Buchs erst beginnen und nicht etwa bereits abgeschlossen werden.

Während sich die Monografie von ihrer Zielsetzung her also als „Einführung“ versteht, weicht sie in ihrer tieferen Struktur durchaus erkennbar von klassischen Einführungswerken oder Lehrbüchern ab. So orientieren sich die dargelegten Überkapitel zwar noch grundsätzlich an einer thematischen Aufteilung, wie sie hierfür

T. Niendorf $(\triangle)$

Institut für Politikwissenschaft, Friedrich-Schiller-Universität Jena, Jena, Deutschland

E-Mail: tim.niendorf@uni-jena.de 
opportun erscheint (Geschichte, Wirtschaft, Innenpolitik/Politische Systeme, Außenpolitik), die jeweiligen Unterkapitel folgen dann aber deutlich stärker argumentativen denn institutionell oder prozessorientierten Kriterien. Ebenso auffällig und auch etwas ungewohnt gestaltet sich der Umgang mit den vorliegenden „Einzelfällen“. So finden sich entgegen vieler klassischer Überblickswerke weder Kapitel zu einzelnen Ländern noch umfangreichere Fallstudien innerhalb der thematischen Kapitel. Stattdessen arbeitet Welz durchgängig mit kurzen Beispielfällen, die jeweils auf ein spezifisches Argument oder konkreten Einzelaspekt (z. B. das Konfliktmanagement der Afrikanischen Union in Mali und der Zentralafrikanischen Republik zur Illustration von Multi-Akteurs-Friedensoperationen) bezogen sind. Dieses Verfahren hat seine Vor-, aber auch stellenweise Nachteile. Zu den offensichtlichen Vorteilen zählt, dass praktisch jeder der 54 (international anerkannten) afrikanischen Staaten zumindest vereinzelt Erwähnung findet. Ebenso ist es hierdurch möglich, die zentrale Zielstellung einer umfangreichen Darstellung der Diversität des Kontinents und seiner Gegensätze in einem überschaubaren Rahmen zu gewährleisten. Allerdings ist es umgekehrt zum einen relativ schwierig (trotz der zahlreichen internen Verweise auf andere Kapitel), die verschiedenen Informationsbausteine, die ein einzelnes Land betreffen, zueinander in Beziehung zu setzen. Andererseits erwartet Leserinnen und Leser, die sich bisher kaum mit Politik in Afrika auseinandergesetzt haben, eine regelrechte Informationsflut an konkreten Beispielen, deren intensiveres Verständnis oftmals das Studium weiterer Literatur erfordert.

Hilfreich für die Nachvollziehbarkeit gestaltet sich aber der konsistente und häufige Rückbezug auf zwei zentrale Narrative bzw. Motive, die durch das Buch leiten. Das erste Motiv ist die bedeutende Rolle von Pfadabhängigkeiten, die sich aus (üblicherweise problematischen) Weichenstellungen während der Kolonialzeit, zum Teil aber auch der vorkolonialen Periode, ergeben. So sind viele der wirtschaftlichen, innerstaatlichen und internationalen Herausforderungen nur unter Rückbezug auf die politischen Entscheidungen und fortwährenden Interessen der ehemaligen Kolonialmächte zu erklären und verstehen. Dies ist im wissenschaftlichen Sinne zunächst eine weder besonders überraschende noch neue Erkenntnis. Es gelingt Welz aber einerseits eindrucksvoll nachzuzeichnen, wie sich die Auswirkungen dieser Weichenstellungen auch in politischen Fragen wiederfinden, die weder einen direkten Bezug zur kolonialen Vergangenheit haben noch von dieser vorgeformt wurden. Anderseits verdeutlicht er intensiv und facettenreich, wie diese direkt und indirekt das Verständnis politischer Realitäten sowohl durch afrikanische als auch nichtafrikanische Akteurinnen und Akteure beeinflussen. Das hieran anknüpfende zweite Motiv findet sich in der bereits erwähnten Gegensätzlichkeit der Interpretationen politischer Entwicklungen auf dem afrikanischen Kontinent, die wiederum zu den von Welz kritisierten Verallgemeinerungen und ,aneinander vorbeireden“ im Dialog mit Afrika führen (S. 236-238). Um dies zu verdeutlichen, führt der Autor immer wieder knappe Überblicke zu relevanten politischen und wissenschaftlichen Diskursen ein, wobei er vermeintlich feststehende Analysekategorien und daraus abgeleitete Fakteninterpretationen hinterfragt (so z. B. die vermeintlich herausgehobene Rolle von Gier und Ethnien als zentrale Konfliktmotivationen oder das widersprüchliche Verhältnis zwischenstaatlicher Solidarität in Afrika) bzw. aufzeigt, wie diese in den betreffenden Diskursen herausgefordert werden. 
Im Sinne der spezifischen Zielsetzung des Buchs ist dieses daher als äußerst gelungener und bereichernder Beitrag zur politikwissenschaftlichen Beschäftigung mit Afrika zu sehen, die insbesondere im deutschsprachigen Raum immer noch einen erkennbaren Mangel an aktuellen Standard- und Überblickswerken aufweist. Das parallele Erscheinen in englischer Sprache bei Cambridge University Press unter dem Titel „Africa Since Decolonization“ wird dabei zweifelsohne zur zusätzlichen wissenschaftlichen Relevanz des Buchs beitragen. Allerdings kann das Buch eben „nur“ eine Einführung sein. Gerade zum umfassenderen Verständnis der zahllosen Einzelbeispiele, aber auch der Vertiefung der gegensätzlichen Interpretationsansätze, die jeweils nur knapp und auf das Wesentliche reduziert präsentiert werden, ist ein weiterführendes Literaturstudium erforderlich. Dies würde jedoch erneut der vom Autor geäußerten Hoffnung einer Anregung zur weiteren Auseinandersetzung mit Afrika entsprechen.

Funding Open Access funding enabled and organized by Projekt DEAL.

Open Access Dieser Artikel wird unter der Creative Commons Namensnennung 4.0 International Lizenz veröffentlicht, welche die Nutzung, Vervielfältigung, Bearbeitung, Verbreitung und Wiedergabe in jeglichem Medium und Format erlaubt, sofern Sie den/die ursprünglichen Autor(en) und die Quelle ordnungsgemäß nennen, einen Link zur Creative Commons Lizenz beifügen und angeben, ob Änderungen vorgenommen wurden.

Die in diesem Artikel enthaltenen Bilder und sonstiges Drittmaterial unterliegen ebenfalls der genannten Creative Commons Lizenz, sofern sich aus der Abbildungslegende nichts anderes ergibt. Sofern das betreffende Material nicht unter der genannten Creative Commons Lizenz steht und die betreffende Handlung nicht nach gesetzlichen Vorschriften erlaubt ist, ist für die oben aufgeführten Weiterverwendungen des Materials die Einwilligung des jeweiligen Rechteinhabers einzuholen.

Weitere Details zur Lizenz entnehmen Sie bitte der Lizenzinformation auf http://creativecommons.org/ licenses/by/4.0/deed.de. 